La Revue

des Droits

de l'Homme

\section{La Revue des droits de l'homme}

Revue du Centre de recherches et d'études sur les droits fondamentaux

Actualités Droits-Libertés | 2016

\title{
Yémen ? Vous avez dit crise humanitaire?
}

Droit international humanitaire et droit international pénal

\section{Jelena Aparac}

\section{OpenEdition}

\section{Journals}

Édition électronique

URL : http://journals.openedition.org/revdh/2545

DOI : $10.4000 /$ revdh. 2545

ISSN : 2264-179X

\section{Éditeur}

Centre de recherches et d'études sur les droits fondamentaux

\section{Référence électronique}

Jelena Aparac, "Yémen? Vous avez dit crise humanitaire ? ", La Revue des droits de l'homme [En ligne], Actualités Droits-Libertés, mis en ligne le 20 mars 2017, consulté le 30 avril 2019. URL : http:// journals.openedition.org/revdh/2545; DOI : 10.4000/revdh.2545

Ce document a été généré automatiquement le 30 avril 2019

Tous droits réservés 


\title{
Yémen? Vous avez dit crise humanitaire?
}

\author{
Droit international humanitaire et droit international pénal
}

Jelena Aparac

1 Sur les 26 millions d'habitants que compte le pays, 18 millions ont besoin d'aide humanitaire. Parmi ceux-ci, 10.3 millions ont besoin d'une assistance urgente, leur vie étant directement en jeu. Ce chiffre représente une augmentation de $15 \%$ depuis le début du conflit en mars $2015^{1}$. Parmi la population, 2.2 millions d'enfants souffrent de malnutrition dont 500.000 de malnutrition aiguë sévère (MAS) ${ }^{2}$. Bien que ces chiffres soient à appréhender avec prudence, notamment en raison de la faible présence d'acteurs humanitaires sur le terrain (d'où la difficulté de collecter des données fiables) ${ }^{3}$, il n'en reste pas moins que le Yémen demeure une urgence humanitaire silencieuse.

2 Le Yémen a connu de nombreux conflits armés jusqu'à aujourd'hui - certains même avant la création du pays ${ }^{4}$. Dès le début 2011, des mouvements de révolte au Yémen se sont accentués en parallèle du mouvement du Printemps Arabe. Des manifestations ont eu lieu contre le chômage, la crise économique et la corruption, mais également en raison de la réforme constitutionnelle visant à permettre au fils du président Saleh d'hériter de la présidence. Pour mettre fin à cette révolte, le gouvernement a recouru à l'usage de la force létale, perdant ainsi le soutien de la communauté internationale. L'ONU a même fait appel à un transfert de pouvoir en octobre 2011. Le 23 novembre 2011, le président Saleh s'est réfugié en Arabie Saoudite voisine.

3 Pour préparer la transition politique, le président Saleh a signé à cette même date le Golf Cooperation Council Initative and the Agreement et a transféré le pouvoir présidentiel à son vice-président devenu président depuis, Monsieur AbdRabbuh Mansur Hadi. L'accord prévoit une solution politique pour mettre fin aux manifestations et une transition en deux phases, dont la première est l'organisation d'élections présidentielles. Ensuite, dans le respect de l'accord, le nouveau gouvernement a mis en place la National Dialogue Conference (NDC) le 18 mars 2013, mandatée pour rédiger la constitution et la soumettre au référendum (prévu en juin 2014). Le mandat de nouveau président Hadi (originairement prévu jusqu'en 2014) a été prolongé sans élection, provoquant une 
offensive des Houthis, un groupe rebelle chiite zaidi, qui a pris le nom de son ancien commandant Hussein Badreddin al-Houthiet. Ils sont arrivés jusqu'à Sana'a, la capitale et s'y sont installés en occupant certains quartiers et créant un comité de révolution. Le référendum n'a jamais eu lieu, et la crise s'est installée, des milliers de personnes descendant de nouveau dans les rues pour exprimer leur colère. Le gouvernement du président Hadi s'est réfugié dans le sud de pays. Le 25 mars 2015, les Houthis ont capturé une base militaire stratégique au nord de la ville portuaire d'Aden ${ }^{5}$. Ce soir-là, le président Hadi a fui à Ryad où il a été reçu par le Ministre de la Défense de l'Arabie Saoudite. Le 26 mars 2015, la Coalition d'Etat dirigée par l'Arabie Saoudite a lancé une opération militaire Decisive Storm (Tempête décisive) avec quatre Etats du Gulf Cooperation Council (Bahreïn, Koweït, Qatar et Emirats Arabes Unis) et quatre Etats de la Ligue Arabe (Egypte, Jordanie, Maroc et Soudan) ${ }^{6}$.

La crise a donné lieu à un conflit de succession, et non pas à une guerre de religions, comme il est parfois mentionné dans certains médias. Il convient d'examiner les éléments permettant la qualification du conflit au Yémen $\left(1^{\circ}\right)$, pour comprendre ensuite la crise humanitaire à long terme $\left(2^{\circ}\right)$.

\section{$1 \%$ - La qualification du conflit armé au Yémen}

6 Le conflit armé au Yémen est complexe. Sa légalité dépend notamment des parties au conflit (A) qui peuvent déterminer la qualification du conflit (B) peut-être même une internalisation du conflit (C). Ces critères amènent vers la question du droit applicable (D).

\section{A - Les parties au conflit}

7 Pour identifier un conflit armé non international il faut en général deux éléments : d'une part, les combats doivent opposer deux ou plusieurs parties au conflit démontrant un certain degré d'organisation, d'autre part, les affrontements doivent avoir un certain degré d'intensité7. Le Yémen est divisé entre le mouvement Houthis d'un côté et le gouvernement de Hadi, internationalement reconnu et soutenu par la Coalition arabe, de l'autre côté.

8 Pour être considérés en tant que partie au conflit, les Houthis doivent avoir un certain degré d'organisation qui se mesure par un faisceau d'indices. Notamment, ils doivent avoir une structure de commandement. Le groupe militaire Houthi, avec sa branche politique Ansar Allah, contrôle militairement et administrativement certains gouvernorats du pays et ont progressivement assumé le contrôle des institutions étatiques ${ }^{8}$. Ils participent également aux pourparlers de paix, démontrant par-là que le groupe est non seulement structuré, mais aussi organisé. Les Houthis ont par ailleurs la capacité de mener des opérations militaires puisqu' en plus d'utiliser des moyens et méthodes de guerre similaires aux forces armées régulières, ils contrôlent en outre des endroits stratégiques. Enfin, le groupe, de par sa structure et par son organisation a sans doute la capacité de respecter et faire respecter le $\mathrm{DIH}^{9}$.

9 Les Houthis sont soutenus par l'ancien président Saleh et partiellement par l'Iran ${ }^{10}$. 

structure et de son organisation. Cependant, un autre élément doit être pris en compte concernant la partie gouvernementale : le rôle et la légitimité du président Hadi. En effet, ce dernier a démissionné durant le printemps $2015^{11}$, mais un mois plus tard il a retiré sa démission suite au soutien apporté par des Etats du Golf et certains chefs tribaux ${ }^{12}$. Néanmoins, c'est depuis son exil qu'il continue de diriger le pays, tant bien que mal. Car, depuis qu'il a quitté Aden en mars 2015, le président a perdu le contrôle sur les services d'administration et de sécurité à travers le Yémen. Certains ministres ont quitté Sana'a alors que le ministre de la défense est détenu incommunicado par les forces Houthis. Le président n'a pas pu assurer la nomination des hauts officiers exerçant leurs fonctions à travers le pays, perdant ainsi le contrôle de la sécurité. En effet, les services administratifs et de sécurité de Sana'a continuent de fonctionner sous le contrôle d'Ansar Allah et le personnel clé reste loyal à l'ancien président Saleh ${ }^{13}$.

11 Toutefois, la communauté internationale reconnait toujours Hadi comme président légitime en exil ${ }^{14}$. Et c'est ce maintien artificiel dans les fonctions présidentielles qui donne sa légitimité à l'intervention militaire de la Coalition. On y reviendra. années.

es Forces de résistance sont des groupes locaux qui reflètent les conditions sociales et politiques de différentes localités. Ils dépendent partiellement du soutien technique et de la formation des conseillers de la Coalition. Ils combattent les Houthis sur plusieurs fronts, sans vraiment être unis entre eux ${ }^{15}$.

14 Al-Qaida et ses groupes affiliés agissent au Yémen depuis 1990. Le phénomène actuel de la « compétition » entre groupes terroristes date de l'émergence d'Ansar al-Sharia en $2009^{16}$. Toutefois, la compétition entre les dirigeants internationaux d'Al-Qaida et ISIL (Daesh) se déplace progressivement d'un niveau local et régional vers un niveau international ${ }^{17}$. A la différence d'ISIS, Al-Qaida dans la péninsule arabe est plus fort en termes de structure, de nombre de combattants et de fréquence des attaques. Al-Qaida au Yémen, qui représente la branche la plus ancienne et la plus dangereuse, selon les Etats Unis, est en opposition avec des Houthis ${ }^{18}$.

On enregistre la présence d'ISIS au Yémen pour la première fois en novembre 2014. Ils s'opposent aux Houthis qu'ils considèrent comme des agents de l'Iran ${ }^{19}$. avec le soutien de pays comme les Etats Unis ${ }^{20}$. L'Arabie Saoudite, mais aussi les Emirats Arabes Unis, ont une relation très ambiguë avec $\mathrm{Al}$ Qaida qui apparemment continuerait de combattre aux côtés de la Coalition contre les Houtis ${ }^{21}$.

\section{B - Un conflit armé non international}

Des nombreux rapports des Nations Unies et des ONG déplorent les moyens et méthodes de combats utilisés ${ }^{22}$, attestant ainsi de l'intensité des combats. Néanmoins, la qualification de conflit armé est, comme cela est le cas la plupart du temps, sensible tant politiquement que diplomatiquement. Cela est particulièrement vrai pour les conflits armés non internationaux qui ne sont règlementés que par l'article 3 commun, le droit international humanitaire coutumier (DIHC) et éventuellement le Protocole Additionnel II, ratifié par Yémen ${ }^{23}$. Or, aucune disposition du droit international humanitaire ne 
définit le conflit armé, aucune autorité n'est habilitée à qualifier le conflit. Ceci constitue un point faible important du DIH.

De prime abord, le conflit semble revêtir un caractère non international. En effet, dans ce conflit, il ne s'agit pas d'affrontements militaires entre des Etats souverains. Il s'agit plutôt du gouvernement d'un Etat souverain se battant contre un groupe armé non étatique ${ }^{24}$ sur son territoire. De plus, d'autres groupes se combattent entre eux. Ce conflit est donc un conflit armé à caractère non international, une guerre civile.

Parallèlement aux conflits armés non internationaux, on peut également constater une multiplication d'interventions multilatérales aux côtés des parties au conflit. Il convient de préciser que, bien que ces interventions soient de plus en plus importantes dans des nouvelles formes de guerres, la doctrine reste toujours divisée sur leur effet sur la qualification du conflit ${ }^{25}$.

\section{C - Internationalisation du conflit?}

20 En vertu de la distinction jus in bello - jus ad bellum, le droit international humanitaire (jus in bello) s'applique au conflit indépendamment de la légitimité de l'intervention militaire des parties eu égard à la Charte des Nations Unies (jus ad bellum) ${ }^{26}$. Cependant, ces deux branches du droit ont des éléments communs : elles nécessitent de connaitre la volonté du gouvernement concernant toute intervention militaire étrangère sur son territoire. Dans un cas, il s'agit de savoir si la Charte des Nations Unies et donc le droit international a été violé, dans l'autre, en droit international humanitaire, la volonté du gouvernement permet de déterminer si le conflit peut être qualifié de conflit international ou non international.

21 Au Yémen, le président Hadi, soutenu par les Nations Unies, a demandé à la Coalition d'intervenir dans un conflit par tout moyen nécessaire ${ }^{27}$. Cette demande officielle, non seulement apporte la légitimité à l'action menée par la Coalition sur le territoire yéménite, mais aussi maintient la qualification de conflit interne pour la simple raison que la Coalition agit sur demande explicite d'un gouvernement d'un Etat souverain ${ }^{28}$. De ce fait, l'intervention militaire étrangère est menée par la Coalition intervenue en soutien d'un gouvernement légitime dans un conflit armé non international préexistant.

La résolution controversée 2216 (2015) du Conseil de Sécurité n'autorise pas explicitement l'intervention de la Coalition. Néanmoins, elle réaffirme le respect de la souveraineté du Yémen et continue de reconnaitre le président en exil (à travers plusieurs paragraphes) dont l'autorité présidentielle peut être mise en doute. Ainsi, puisque le gouvernement yéménite « invite » officiellement l'Arabie Saoudite à intervenir militairement sur son territoire pour combattre les Houthis, il était important d'affirmer la légitimité du président yéménite, et par conséquent de sa demande. Tel était l'objectif principal de la résolution 2216.

L'intensité de l'usage de la force employée par la Coalition ne sème aucun doute sur sa participation au conflit. Car, seuls des faits réels et objectifs, donc la nature de l'engament de la Coalition permettent d'établir son implication dans un CANI préexistant ${ }^{29}$. La Coalition est donc une partie au conflit. Son intervention apporte un important soutien aux forces gouvernementales.

L'internationalisation peut transformer la nature du conflit si un Etat étranger intervient en soutien à des groupes armés non étatiques, ici les Houthis. Il a été rapporté que l'Iran 
apporte un soutien logistique et technique aux Houthis. En effet, le Panel d'expert du Comité de Sanction 2140 concernant le Yémen a précisé que l'Iran a peut-être envoyé de petites expéditions d'armes. Toutefois, le Panel n'a pas trouvé des éléments pour confirmer que l'exportation d'armes vers le Yémen se produit à grand échelle. Plutôt, et contrairement ce qui a été rapporté par les média, les armes saisies en 2015 étaient sans doute destinées à la Somalie ${ }^{30}$.

Les deux branches du droit international que nous évoquions ont un autre élément commun : la nécessité de déterminer la nature du soutien apporté par une tierce partie à l'un des protagonistes non étatiques au conflit préexistant. Le CICR a récemment fait un état des lieux des différentes interprétations de ce type de soutien. Dans son nouveau positionnement, le CICR distingue le soutien aux parties au conflit tel qu'interprété par le droit de la responsabilité internationale des Etats et le soutien aux parties au conflit préexistant afin de qualifier le conflit au sens du DIH $^{31}$. Cette position s'aligne avec la dernière jurisprudence de la Cour internationale de la justice en la matière et tranche définitivement la question du critère de contrôle global et le critère de contrôle effectif ${ }^{32}$ en faveur de ce premier ${ }^{33}$. Or, même les critères du contrôle global ne semblent pas être satisfaits en l'espèce, car l'Iran, selon le rapport de Panel d'experts, n'apporte qu'un faible soutien aux Houthis.

En outre, toujours selon la position officielle du CICR récemment révisée, la notion de conflit armé interne internationalisé doit être abandonnée par le CICR car il emporte confusion sur le caractère du confliti ${ }^{34}$. En effet, selon le CICR, cette qualification suggère une fausse application du DIH applicable en conflit armé international puisqu'elle est en contradiction avec son approche de fragmenter le conflit selon les parties impliquées.

\section{D - Le droit applicable}

27 Selon l'approche traditionnelle du CICR, confirmée dans un document de position sur la qualification des conflits armés, le droit applicable doit suivre une approche fragmentée, à savoir à chaque relation bilatérale s'applique le droit applicable à ce type de conflit ${ }^{35}$. Bien que cette approche rencontre des critiques $^{36}$ et obstacles réels en pratique (notamment pour des acteurs humanitaires), les Etats ont tendance à accepter cette approche fragmentée par peur de légitimer les groupes armés non étatiques.

Le droit applicable dans le conflit au Yémen où la Coalition intervient en soutien militaire auprès d'une partie étatique, à la demande du gouvernement, est le droit du conflit armé non international.

Il est important de souligner ici que le DIH ne s'applique pas uniquement aux parties au conflit stricto sensu, mais aussi à la Coalition multinationale qui soutient les forces gouvernementales ${ }^{37}$, dès lors qu'elle recourt à l'usage de la force armée en rapport avec le conflit amés ${ }^{38}$. Ainsi, le droit du CANI s'applique aussi bien à la relation Coalition- Houthis qu'à la relation force armée gouvernementale- Houthis ${ }^{39}$. Le droit applicable est le droit international humanitaire applicable en conflit armé non international, à savoir, l'article 3 commun. Le groupe rebelle Houthis, partie au conflit, contrôle plusieurs gouvernorats du territoire du sud du Yémen. Ce groupe combat les forces armées de l'Etat. Enfin, le Yémen a ratifié le Protocole Additionnel II en 1990. Ainsi, tous les critères de l'applicabilité de ces textes sont remplis. 
Le droit applicable dans les relations entre les groupes armés organisés demeure l'article 3 commun et le droit international humanitaire coutumier ${ }^{40}$.

Nonobstant des obligations internationales qui pèsent sur les parties aux conflits, le contexte au Yémen présente une crise humanitaire qui va s'étendre dans le temps.

\section{$2 \%$ Une crise humanitaire de longue durée}

2 La crise humanitaire au Yémen est marquée par les violations répétitives des règles applicables à la conduite des hostilités (A), qui a un impact direct sur des civils (B). Malheureusement, l'enquête pour violations du DIH et des droits de l'Homme risque de ne pas soulever les responsabilités juridiques (C).

\section{A - Les moyens et des méthodes de guerre violant le DIH}

3 Dans un conflit armé, le droit des parties au conflit de choisir les méthodes ou moyens de guerre n'est pas illimitée ${ }^{41}$. En effet, dans leur conduite des hostilités, les parties au conflit, y compris la Coalition, ont des obligations issues du DIHC, telles que l'obligation de respecter le principe fondamental de distinction entre civils et combattants. De plus, ils ont une obligation de précaution avant de lancer des attaques, mais aussi d'utiliser des moyens et méthodes provoquant le moins de dommages possibles, dans le respect du principe du plus petit dommage. Il pèse sur les parties une interdiction d'utiliser des armes indiscriminées par nature qui viole le principe fondamental de distinction entre civils et combattants. Selon le rapport du Panel d'experts, toutes les parties au conflit ont violé les principes susmentionnés, y compris par usage d'armes lourdes ${ }^{42}$, notamment en attaquant des biens civils dans les quartiers résidentiels ${ }^{43}$. En outre, les parties, notamment la Coalition a bombardé à de nombreuses reprises les hôpitaux, infrastructure sanitaire et des écoles, tous étant des biens civils ${ }^{44}$.

Les Houthis, ainsi que la Coalition ont été accusé d'utilisation de moyens de guerre interdits par le DIH, notamment des mines anti-personnelles prohibées par la Convention d'Oslo de $1997^{45}$. Les parties ont également été accusées d'usage d'armes à sous-munition ${ }^{46}$ , bannies par la Convention de Dublin du 30 mai 2008.

De plus, les parties au conflit ont utilisé des méthodes interdites. A cet égard, les Houthis ont été accusés d'utiliser des boucliers humains, y compris des réfugiés ${ }^{47}$. La famine comme méthode de guerre, interdite par la règle 53 du DIHC, est la conséquence directe d'actions des Houthis et de la Coalition.

Le pays souffre aussi d'un blocus de facto. La résolution 2216 (2015) impose un embargo sur les armes, le soutien financier et toute autre forme de soutien militaire et paramilitaire (uniquement) envers les Houthis. De ce fait, les cargos à la destination du Yémen doivent subir des contrôles afin de respecter la résolution susmentionnée. Ces contraintes réelles ont un impact direct sur la situation économique du pays. En effet, vider des cargos peut prendre jusqu'à 30 jours en raison du mauvais management des ports et des obstacles politiques et bureaucratiques, ralentissant ainsi d'autant la distribution des biens à la population ${ }^{48}$. En outre, la Coalition a suspendu tous les vols commerciaux en aout 2016 vers Sana'a, la capitale ${ }^{49}$. La suspension des vols commerciaux a des conséquences directes pour des personnes en situation de besoin médical d'évacuation et empêche environ 20000 personnes d'accéder à des soins médicaux vitaux 
${ }^{50}$. Bien avant le conflit, la majorité des besoins des yéménites était assurée à travers des échanges commerciaux. Aujourd'hui, le pays, où $90 \%$ de l'alimentation et $100 \%$ des médicaments doivent être importés, souffre d'une incapacité logistique de prendre en charge des civils.

\section{B - Les civils au Yémen, victimes directes d'une catastrophe humanitaire oubliée}

De manière générale, le système des Nations Unies apporte une pauvre réponse à la crise humanitaire au Yémen. L'OCHA ne briffe qu'occasionnellement le Conseil de sécurité, tandis que les stratégies opérationnelles des acteurs humanitaires ne répondent pas entièrement aux besoins humanitaires ${ }^{51}$.

\section{1) Accès humanitaire}

Les parties au conflit doivent autoriser et faciliter le passage rapide et sans encombre de secours humanitaires destinés aux personnes civiles dans le besoin ${ }^{52}$. Or, la question d'accès humanitaire est une question complexe. En réalité, l'accès vers la population est possible. Les ONG peuvent obtenir des visas et des autorisations nécessaires pour se rendre au Yémen ${ }^{53}$. Toutefois, la sécurité demeure un véritable obstacle ${ }^{54}$. Les ONG sont suivies par les Houthis qui se montrent méfiants envers les organisations étrangères ${ }^{55}$. En outre, des acteurs humanitaires ont été kidnappés, détenus illégalement et tués lors de leurs opérations de secours ${ }^{56}$. Ceci explique également une faible présence des ONG internationales, notamment de leur personnel expatrié ${ }^{57}$. Sur 114 organisations présentes au Yémen, seules 23 sont des organisations non gouvernementales internationales (IONG) et 8 agences des Nations Unies En comparaison, à la même période, en Afghanistan, l'OCHA a enregistré 172 organisations présentes ${ }^{58}$. A cet aspect dangereux de conflit s'ajoute une gestion complexe de la sécurité, y compris la nécessité d'établir et de maintenir des contacts avec les parties aux conflits ${ }^{59}$.

\section{2) Accès à la santé}

Outre le fait que les civils sont des victimes directes dans la majorité des attaques, la population civile est aussi une victime indirecte notamment depuis la fermeture de l'aéroport de Sana'a aux vols commerciaux ${ }^{60}$.Ceci contribue également à la destruction progressive du système de santé du pays, alors que le secteur de santé était déjà largement dysfonctionnel avant même le début du conflit ${ }^{61}$. En effet, seulement $45 \%$ de l'infrastructure sanitaire fonctionne avec une capacité réduite, notamment en raison d'absence d'essence, des ressources et de personnel médical. Aujourd'hui, selon MSF "plus de 100 structures sanitaires ont été partiellement ou entièrement détruites, 600 autres sont non fonctionnelles $»^{62}$. Depuis la fermeture de l'aéroport, la population meurt des pathologies préventives. Les soins de santé primaire dépendent entièrement de l'aide étrangère ${ }^{63}$. Le Yémen, qui se trouve de plus en plus isolé, souffre de la crise économique qui affecte le peu de ce qui reste du système de santé dans le pays 


\section{3) Refugiés et déplacés internes}

40 Le conflit continue de provoquer des déplacements de population sans précédent ${ }^{64}$. Selon le Haut-Commissariat des Nations Unies pour les réfugiés (UNHCR), 180992 des personnes de nationalité yéménite sont déplacées dans la région ${ }^{65}$. Les principaux pays d'accueil sont Oman, l'Arabie Saoudite, Djibouti et la Somalie. Mais selon l'ONU, 107000 migrants et refugiés sont arrivé au Yémen en 2016 malgré le conflit, dans l'espoir de s'installer dans des pays du Golfe ${ }^{66}$.

41 Parmi les 3.11 millions des personnes déplacées à l'intérieur du pays (IDPs) ${ }^{67}$, il est estimé que $52 \%$ sont des femmes dont $22 \%$ des filles ${ }^{68}$. Selon le Fond des Nations Unies pour la Population (UNFPA) presque 3 millions de femmes et filles risquent de subir des violences sexuelles basées sur le genre ${ }^{69}$.

\section{4) Enfants}

42 Le Rapport du Secrétaire général des Nations Unies sur les enfants et les conflits armés a donné une liste des parties au conflit qui « recrutent, tuent, violent ou commettent tout autre type de violence sexuelle, attaquent des écoles et des hôpitaux ou enlèvent les enfants ». Il a constaté que toutes les parties au conflit au Yémen sont responsables de tels actes envers des enfants, y compris l'Arabie Saoudite ${ }^{70}$. Suite aux menaces de l'Arabie Saoudite, l'annexe du rapport a été modifiée pour le retirer de la liste ${ }^{71}$.

\section{C - Les enquêtes et poursuites pour des violations du droit international humanitaire et des droits de l'Homme}

Une nouvelle résolution du Conseil de sécurité qui est en discussion depuis des mois (mais en stand by pour permettre aux pourparlers de paix d'avancer) devrait renforcer les aspects de conduite des hostilités et d'accès humanitaire, notamment la sécurité. Néanmoins, le cadre actuel de médiation ne permet pas de faire avancer les accords de paix, qui doit avant tout réunir des conditions d'une paix durable. Parmi ces conditions, il faudra préparer la transition politique pour permettre d'intégrer l'ensemble de la population dans les institutions politiques du pays. Or, les parties au conflit sont en déséquilibre complet, même à travers ce qui est compris dans le texte de la résolution 2216 (2015).

De plus, la transition vers la paix passe également par la réparation et la lutte contre l'impunité. Or, là encore, le Yémen est dans l'impasse. En effet, dès septembre 2015 le Haut-Commissariat des Nations Unies aux droits de l'Homme a proposé d'établir une commission d'enquête internationale d'établissement des faits ${ }^{72}$, un mécanisme ad hoc modelé sur d'autres commissions de ce type, comme en Syrie par exemple. Mais suite à l'opposition forte des pays arabes, le Conseil de droit de l'Homme de l'ONU a dû se contenter uniquement d'une Commission nationale d'enquête avec le soutien de l'équipe technique des Nations Unies qui doit rapporter oralement lors de chaque session du Conseil ${ }^{73}$.

Bien que cela puisse paraitre comme un progrès dans la documentation des violences commises au Yémen, les conclusions du Joint Incidents Assessment Team (JIAT) mise en 
place par la Coalition suite à des attaques sur des biens civils, diffèrent des rapports des Nations Unies et de Human Rights Watch ${ }^{74}$. En outre, la Commission nationale a été accusée de ne pas respecter son mandat selon les « standards internationaux » ${ }^{75}$.

Le Haut-Commissariat pour les droits de l'homme a réitéré son appel à la création d'une commission internationale indépendante d'enquête en août $2016^{76}$. Bien que les Houthis paraissent favorable à la mise en place d'une telle commission internationale d'enquête ${ }^{77}$, cette perspective est politiquement inacceptable pour la France et les Etats Unies qui ont des liens forts avec l'Arabie Saoudite, notamment depuis les signatures des importants contrats de vente d'armes. Enfin, il faut noter que le Yémen n'est pas un Etat partie au Statut de Rome et par conséquent, la Cour pénale internationale (CPI) ne peut pas poursuivre les violations graves du DIH sur son territoire. Seule la résolution du Conseil de sécurité pour référer l'affaire à la CPI. Or, c'est une évidence, les intérêts des membres $\mathrm{du}$ Conseil sont en contradiction avec une telle résolution.

Le conflit pousse le pays déjà pauvre vers un effondrement institutionnel, social et économique ${ }^{78}$. Le Yémen est en train de devenir un failed state (Etat failli) avec un système d'impunité instauré.

Les Lettres « Actualités Droits-Libertés » (ADL) du CREDOF (pour s'y abonner et se désabonner) sont accessibles sur le site de la Revue des Droits de l'Homme (RevDH) Contact

\section{NOTES}

1. OCHA, Humanitarian Bulletin, no. 18, 14 décembre 2016.

2. Idem. Selon UNICEF 323000 enfants de moins de cinq ans sont affectés par MAS https:// www.unicef.org/appeals/yemen.html\#10 (visité le 31 janvier 2017).

3. Voir, OCHA, YEMEN : Organizations $3 W$ Operational Presence, 30 novembre 2016, disponible ne ligne : http://reliefweb.int/sites/reliefweb.int/files/resources/yemen_3w_nov_13_2016_en.pdf (visité le 31 janvier 2017).

4. En effet, des guerres civiles ont eu lieu Yémen du nord (1962-1970) ou au Yémen du sud avec la fin de la domination britannique (1963-1967) puis ont été remplacés par un conflit interétatique entre les deux Yémen (1972) jusqu'à leur réunification en 1990. Bien que formé par les représentants des deux gouvernements, le nouveau Yémen avec une domination forte du nord n'a cessé de marginaliser économiquement le sud du pays. De plus, les deux armées n'ont jamais fusionnées pour former une seule force armée, favorisant la poursuite d'affrontements internes jusqu'à la défaite des sécessionnistes de sud et du parti Socialiste di Yémen exilé en Arabie Saoudite. En outre, le président de l'époque se montrait particulièrement coopératif à l'égard des Etats Unis notamment dans la " guerre contre le terrorisme » suite aux attentats de New York de 11 septembre 2001.

5. International Crisis Group, Yemen at war, Briefing no. 45, 28 March 2015. 
6. Letter dated 22 January 2016 from the Panel of Experts on Yemen established pursuant to Security Council resolution 2140 (2014) S/2016/73, addressed to the President of the Security Council, 26 janvier 2016, p. 12, para. 27.

7. Voir l'article 3 commun aux quatre Conventions de Genève du 12 août 1949, ainsi que l'article 1 du Protocole additionnel II du 8 juin 1977, Procureur c. Dusko Tadic, TPIY, affaire n IT-94-1-AR72, Chambre d'appel, arrêt relatif à l'appel de la défense concernant l'exception préjudicielle d'incompétence, 2 octobre 1995, § 70 ; Procureur c. Haradinaj, TPIY, affaire $\mathrm{n}^{\circ}$ IT-04-84-T, jugement en date du 3 avril 2008, par. 60 ; Procureur c. Ljube Boskovski et Johan Tarculovski, TPIY, affaire No. IT-04-82-T, jugement, 10 juillet 2008, para. 176.

8. Dès mai 2012, les Houthis ont pris le contrôle de plusieurs gouvernorats, notamment le gouvernorat de Saada et une partie d 'Amran, Al Jawf et Hajjah ce qui leur a permis l'accès vers la Mer Rouge ; v. aussi Letter dated 22 January 2016 from the Panel of Experts on Yemen established pursuant to Security Council resolution 2140 (2014) , S/2016/73, addressed to the President of the Security Council, 26 janvier 2016, p. 10, para. 1

9. Idem, pp. 15-18.

10. New York Times, Plight of Houthi Rebels Is Clear in Visit to Yemen's Capital, 26 novembre 2016 disponible en ligne: https://www.nytimes.com/2016/11/26/world/middleeast/houthi-rebelsyemen.html (visité le 24 janvier 2017).

11. Voir http://english.alarabiya.net/en/News/middle-east/2015/01/22/Yemen-governmentoffers-resignation.html; http://edition.cnn.com/2015/01/22/world/yemen-violence/ (visité le 29 janvier 2017).

12. Voir http://www.aljazeera.com/news/2015/02/yemen-abd-rabbu-masnourhadi-150224001628332.html (visité le 29 janvier 2017).

13. Letter dated 22 January 2016 from the Panel of Experts on Yemen established pursuant to Security Council resolution 2140 (2014),S/2016/73, addressed to the President of the Security Council, 26 janvier 2016, p. 12, para 29.

14. Conseil de sécurité, résolution 2216 en date du 14 avril 2015, S/RES/2216 (2015).

15. Letter dated 22 January 2016 from the Panel of Experts on Yemen established pursuant to Security Council resolution 2140 (2014), S/2016/73, addressed to the President of the Security Council, 26 janvier 2016, p. 17, para. 50.

16. Idem, p. 18, para. 54 .

17. Idem, p. 19, para 55.

18. Idem, p. 19, para. 58.

19. Idem, p. 19, para. 56.

20. Voir le communiqué de presse de Ministère de la défense des Etats Unis en date du 29 janvier 2017 : https://www.defense.gov/News/Article/Article/1063593/us-forces-kill-14-al-qaida-in-thearabian-peninsula-terrorists-in-yemen (visité le 30 janvier 2017) ; http://www.aljazeera.com/ news/2017/01/yemen-170129101045539.html (visité le 30 janvier 2017).A noter qu'Al Qaida apport le soutien à la Coalition mais il a été rapporté que l'ISIS aussi.

21. Entretien avec Laurent Sury, chargé du desk urgence de MSF France, le 31 janvier 2017. Selon M. Sury, il a été rapporté qu'ISIS a aussi combattu pour la Coalition contre les Houthis.

22. Voir le briefing au Conseil de sécurité par l'envoyé spécial pour le Yémen Ismail Ould Cheikh Ahmed, le directeur de l'OCHA Stephen O’Brien et le directeur régional du Programme Mondial d'Alimentation, Muhannad Hadi, 31 octobre 2016, S/PV.7797 ; OCHA, Humanitarian Bulletin, no 18, décembre 2016 ;Human Rights Watch, https://www.hrw.org/world-report/2017/countrychapters/yemen (visité le 30 janvier 2017).

23. Article 3 commun aux quatre Convention de Genève du 12 aout 1949 ; HENCKAERTS J.-M. et DOSWALD-BECK L., Customary international humanitarian law, Cambridge, Cambridge UP, 2005, 2 vol. (vol. 1 : Rules, vol. 2 : Practice), p. 674 et p. 4545. Protocole additionnel aux Conventions de 
Genève du 12 août 1949 relatif à la protection des victimes des conflits armés non internationaux (Protocole II), 8 juin 1977.

24. Voir l'article 3 commun aux quatre Conventions de Genève de 1949, article $1 \mathrm{du}$ Protocol additionnel II de 1977, Procureur c. Dusko Tadic, TPIY, affaire n IT-94-1-AR72, Chambre d'appel, arrêt relatif à l'appel de la défense concernant l'exception préjudicielle d'incompétence, 2 octobre 1995, § 70 ; Procureur c. Haradinaj, TPIY, affaire $n^{\circ}$ IT-04-84-T, jugement en date du 3 avril 2008, par. 60.

25. FERRARO T., «Applicabilité et application du droit international humanitaire aux forces multinationales ", Volume 95 Sélection française 2013 / 3 et 4, pp. 71-125.

26. Voir notamment l'article 1 et 2 commun aux quatre Convention de Genève, le préambule du Protocole Additionnel I « Réaffirmant, en outre, que les dispositions des Conventions de Genève du 12 août 1949 et du présent Protocole doivent être pleinement appliquées en toutes circonstances à toutes les personnes protégées par ces instruments, sans aucune distinction défavorable fondée sur la nature ou l'origine du conflit armé ou sur les causes soutenues par les Parties au confit, ou attribuées à celles-ci ».

27. Conseil de sécurité, résolution 2216, en date du 14 avril 2015, S/RES/2216 (2015), alinéa 2.

28. Si la communauté internationale ne reconnaitra pas le président Hadi comme légitime, l'intervention Saoudienne contre les Houthis qui contrôlent (effectivement) une partie du territoire pourra être considérée comme une violation de la Charte des Nations Unies, mais aussi comme un conflit opposant une Coalition et un autre Etat, et donc pourrait être qualifié de conflit armé international.

29. Selon le CICR, la partie tierce qui apporte son soutien à l'un de belligérant peut être vue comme une partie au conflit si elle remplit les quatre conditions suivantes : 1) il existe un CANI sur le territoire duquel la tierce partie intervient ; 2) la tierce partie entreprend des actions relatives à la conduite des hostilités dans le contexte du CANI préexistant ; 3) l'intervention militaire de la tierce partie soutient l'un des belligérants au CANI préexistant ; 4) l'action par la tierce partie est mise en œuvre suite à une décision officielle propre dont l'objectif est de soutenir une partie au conflit préexistant ; FERRARO T., "The ICRC's legal position on the notion of armed conflict involving foreign intervention and on determining the IHL applicable to this type of conflict", RICR (2015), 97 (900), p. 1231.

30. Voir http://www.whatsinblue.org/2017/01/yemen-briefing-on-political-and-humanitariandevelopments-and-2140-committee-meeting.php\#read-more (visité le 27 janvier 2017). Et surtout: Letter dated 27 January 2017 from the Panel of Experts on Yemen addressed to the President of the Security Council, 31 janvier 2017, S/2017/81..

31. FERRARO T., "The ICRC's legal position on the notion of armed conflict involving foreign intervention and on determining the IHL applicable to this type of conflict", RICR (2015), 97 (900), p. 1231. CIJ, affaire relative à l'application de la convention pour la prévention et la répression du crime de génocide (Bosnie-Herzégovine c. Serbie et Monténégro), arrêt du 26 février 2007 (fond), para. 404.

32. $\mathrm{CIJ}$, affaire relative à l'application de la convention pour la prévention et la répression du crime de génocide (Bosnie-Herzégovine c. Serbie et Monténégro), arrêt du 26 février 2007 (fond), para. 404. Voir aussi, FERRARO T, "The ICRC's legal position on the notion of armed conflict involving foreign intervention and on determining the IHL applicable to this type of conflict", pp. 1234-1240.

33. "Pour imputer la responsabilité d'actes commis par des groupes militaires ou paramilitaires à un État, il faut établir que ce dernier exerce un contrôle global sur le groupe, non seulement en l'équipant et le finançant, mais également en coordonnant ou en prêtant son concours à la planification d'ensemble de ses activités militaires. Ce n'est qu'à cette condition que la 
responsabilité internationale de l'État pourra être engagée à raison des agissements illégaux du groupe. Il n'est cependant pas nécessaire d'exiger de plus que l'État ait donné, soit au chef du groupe soit à ses membres, des instructions ou directives pour commettre certains actes spécifiques contraires au droit international. », Procureur c. Dusko Tadic, TPIY, affaire No. IT-94-1A, Chambre d'appel, jugement, 15 juillet 1999, para. 131.

34. FERRARO T., "The ICRC's legal position on the notion of armed conflict involving foreign intervention and on determining the IHL applicable to this type of conflict", p. 1230.

35. Idem. pp. 1240-1243.

36. Procureur c. Dusko Tadic, TPIY, affaire No. IT-94-1-T, Chambre d'appel, arrêt relatif à l'appel de la défense concernant l'exception préjudicielle d'incompétence, 2 octobre 1995, opinion dissidente du juge Li, para. 7 ; MERON T, "Classification of Armed Conflict in the Former Yugoslavia: Nicaragua's Fallout", American Journal of International Law, vol. 92, No. 2, 1998, pp. 236-238.

37. FERRARO T., «Applicabilité et application du droit international humanitaire aux forces multinationales », pp. 71-125.

38. Idem, p. 89 : «tout recours à la force armée par les forces multinationales en rapport avec les hostilités qui ont lieu pendant le conflit armé sera soumis aux exigences du DIH, que ces forces agissent ou non en situation de légitime défense. ".

39. Voir FERRRO T. The ICRC's legal position on the notion of armed conflict involving foreign intervention and on determining the IHL applicable to this type of conflict", pp. 1243-1245.

40. Par l'effet de l'article 1 du Protocol Additionnel II, ce traité ne s'applique pas entre les deux groupes armés non étatiques.

41. Article 22 du Règlement de La Haye de 1907. Le règlement a le caractère coutumier.

42. Les Houthis sont entrés en possession d'armes lourdes avec la prise de contrôle des institutions de sécurité dans les différentes localités.

43. Letter dated 22 January 2016 from the Panel of Experts on Yemen established pursuant to Security Council resolution 2140 (2014), S/2016/73, addressed to the President of the Security Council, 26 janvier 2016, p. 36, para. 124; Human Righst Watch, Yemen- events of 2016, disponible en ligne: https://www.hrw.org/world-report/2017/country-chapters/yemen\#cc98ff (visité le 30 janvier 2017) ; Selon les Médecins Sans Frontières (MSF), la Coalition a bombardé des biens civils tels que le camp de déplacés d'El-Mazraa, dans le gouvernorat de Hajjah, un camionciterne, un marché en pleine activité au moment de la rupture du jeûne du ramadan bombardé à plusieurs reprise. Selon le MSF, Les Houthis ont commis des «bombardent aveuglément des zones résidentielles densément peuplées ». TERZIAN M., Yémen : crimes de guerre et pénuries extrêmes, 27 juillet 2015, disponible en ligne : http://www.msf.fr/actualite/publications/yemencrimes-guerre-et-penuries-extremes (visité le 30 janvier 2017); Voir MSF, dossier Yémen, disponible ne ligne: http://www.msf.fr/actualite/dossiers/crise-au-yemen (visité le 30 janvier 2017).

44. Letter dated 22 January 2016 from the Panel of Experts on Yemen established pursuant to Security Council resolution 2140 (2014), S/2016/73, addressed to the President of the Security Council, 26 janvier 2016, paras. 133-138; Voir briefing au Conseil de sécurité par l'envoie spécial pour Yémen Ismail Ould Cheikh Ahmed, le directeur de l'OCHA Stephen O'Brien et le directeur régional du Programme Mondial d'Alimentation, Muhannad Hadi, 31 octobre 2016, S/PV.7797 ; v. aussi Saudi-led coalition strikes Yemeni port, civilians at risk disponible en ligne http:// www.reuters.com/article/us-yemen-security-idUSKBN15P12V (visité le 10 février 2017).

45. Le Yémen a ratifié la Convention le 1er septembre 1998, elle a entrée en vigueur le mars 1999 pour celui-ci.

46. Human Rights Watch, Yemen-events of 2016, disponible en ligne : https://www.hrw.org/ world-report/2017/country-chapters/yemen\#cc98ff (visité le 30 janvier 2017). Toutefois, le Yémen, l'Arabie Saoudite et les autres membres de la Coalition ne sont pas des Etats membres à la 
Convention sur l'interdiction des bombes à sous munition de 2008. Ils sont toutefois liés par des principes humanitaires reconnus comme coutumier.

47. Letter dated 22 January 2016 from the Panel of Experts on Yemen established pursuant to Security Council resolution 2140 (2014), S/2016/73, addressed to the President of the Security Council, 26 janvier 2016, p. 38, para. 133.

48. Voir le briefing au Conseil de sécurité par l'envoyé spécial pour le Yémen Ismail Ould Cheikh Ahmed, le directeur de l'OCHA Stephen O’Brien et le directeur régional du Programme Mondial d'Alimentation, Muhannad Hadi, 31 octobre 2016, S/PV.7797, p. 6 ; v. aussi Saudi-led coalition strikes Yemeni port, civilians at risk disponible en ligne http://www.reuters.com/article/usyemen-security-idUSKBN15P12V (visité le 10 février 2017).

49. Voir Statement of the Humanitarian Coordinator in Yemen, Jamie McGoldrick, on the Continued Violations of the Protection of Civilians, 29 août 2016.

50. OCHA, Humanitarian Bulletin, no. 19, 31 décembre 2016.

51. Voir le site de MSF France, dossier Yémen, disponible en ligne : http://www.msf.fr/pays/ yemen (visité le 31 janvier 2017).

52. Règle 55 du DIHC.

53. Entretien avec Laurent Sury, chargé du desk urgence de MSF France, le 31 janvier 2017.

54. Office of the Humanitarian Coordinator in Yemen, Humanitarian Coordinator in Yemen, Jamie McGoldrick, Statement on the situation in the Dhubab and Al Mokha Areas, Sana'a, 31 janvier 2017.

55. Entretien avec Laurent Sury, chargé du desk urgence de MSF France, le 31 janvier 2017.

56. HumanRights Watch, Yemen - events; Voir briefing au Conseil de sécurité par l'envoie spécial pour Yémen Ismail Ould Cheikh Ahmed, le directeur de l'OCHA Stephen O'Brien et le directeur régional du Programme Mondial d'Alimentation, Muhannad Hadi, 31 octobre 2016, S/PV.7797, p. 5.

57. Entretien avec Laurent Sury, chargé du desk urgence de MSF France, le 31 janvier 2017 ; OCHA, YEMEN : Organizations 3W Operational Presence, 30 novembre 2016.

58. Voir Reliefweb, Afghanistan : Humanitarian Operational Presence (3W) http://reliefweb.int/ map/afghanistan/afghanistan-humanitarian-operational-presence-3w-october-december-2016 (visité le 31 janvier 2017).

59. Entretien avec Laurent Sury, chargé du desk urgence de MSF France, le 31 janvier 2017.

60. Report from UN Office for the Coordination of Humanitarian Affairs, UN Humanitarian Coordinator in Yemen du 29 août 2016; Office of the Humanitarian Coordinator in Yemen, Humanitarian Coordinator in Yemen, Jamie McGoldrick, Statement on the situation in the Dhubab and Al Mokha Areas, Sana'a, 31 janvier 2017.

61. HumanRights Watch, Yemen-events ; Voir briefing au Conseil de sécurité par l'envoie spécial pour Yémen Ismail Ould Cheikh Ahmed, le directeur de l'OCHA Stephen O'Brien et le directeur régional du Programme Mondial d'Alimentation, Muhannad Hadi, 31 octobre 2016, S/PV.7797, p. 5 .

62. Voir le site de MSF France, dossier Yémen, disponible en ligne : http://www.msf.fr/pays/ yemen (visité le 31 janvier 2017).

63. OCHA, Humanitarian Bulletin, no. 19, 31 décembre 2016.

64. Conseil de droit de l'homme, Situation of human rights in Yemen Report of the United Nations High Commissioner for Human Rights, 4 août 2016, A/HRC/33/38, para. 64.

65. Yemen Situation : Regional Refugee and Migrant Response - Population movement out of Yemen, au 31 octobre 2016.

66. Voir l'Organisation international de migration (OIM), https://www.iom.int/countries/yemen (visité le 31 janvier 2017).

67. OCHA, 2017 YEMEN Humanitarian Response Plan (YHRP), key figures; disponible en ligne http:// www.unocha.org/yemen, visité le 10 février 2017. 
68. OCHA, Humanitarian Bulletin, no. 19, 14 décembre 2016.

69. Idem.

70. Report of the Secretary-General, A/70/836-S/2016/360, 20 avril 2016, annex I List of parties that recruit or use children, kill or maim children, commit rape and other forms of sexual violence against children, or engage in attacks on schools and/or hospitals, or abduct children in situations of armed conflict on the agenda of the Security Council, p. 39.

71. Children and armed conflict, Report of the Secretary-General, A/70/836/Add.1-S/2016/360/ Add.1, 24 juin 2016.

72. Conseil des droits de l'Homme, Situation of human rights in Yemen, Report of the United Nations High Commissioner for Human Rights, 7 septembre 2015, A/HRC/30/31, para. 91.

73. Conseil des droits de l'Homme, Resolution adopted by the Human Rights Council on 2 October 2015 30/18.Technical assistance and capacity-building for Yemen in the field of human rights, 12 octobre 2015, A/HRC/RES/30/18.

74. Human Rights Watch, Yemen - events.

75. Idem.

76. Conseil des droits de l'Homme, Situation of human rights in Yemen, Report of the United Nations High Commissioner for Human Rights, 4 août 2016, A/HRC/33/38, para. 74.

77. V. Yemen rebels call on U.N. to investigate bombings, dismiss peace envoy http:// www.reuters.com/article/us-yemen-security-un-idUSKBN15P2IZ (visité le 11 février 2017).

78. OCHA, Humanitarian Bulletin, no. 19, 31 décembre 2016.

\section{RÉSUMÉS}

Le conflit armé au Yémen a été déclenché en mars 2015 par une intervention militaire de la Coalition de plusieurs pays du Golf et de la Ligue Arabe à la demande du président Hadi en exil à Riyad. La Coalition, qui agit sur commandement militaire de l'Arabie Saoudite, combat le groupe armé Houthi qui contrôle militairement et administrativement une partie du territoire ensemble avec sa branche politique Ansar Allah. Les Houthis sont soutenus par l'ancien président Saleh. Le conflit soulève des questions juridiques importantes telles que la qualification du conflit armé au Yémen ainsi que son éventuel internationalisation et le droit applicable. En outre, le conflit s'inscrit dans un contexte économique et politique désastreux et aggrave la situation humanitaire du pays. En effet, sur 26 millions d'habitants, 18 sont en grande situation de besoin humanitaire et parmi ceux-ci, 10.3 million sont dans un besoin urgent d'aide humanitaire, sans laquelle ils risquent leurs vies. Ce drame interroge aussi sur l'absence de couverture médiatique alors que les personnes et des biens civils sont les premiers à être touchés par des attaques militaires ciblées, en violation du droit international humanitaire. Malgré cela, il n'y aura aucune enquête internationale indépendante et impartiale pour documenter les violences. Le Yémen est en train de devenir un « failed state ».

\section{INDEX}

Mots-clés : Conflit armé, conflit interne, conflit interne internationalisé, crise humanitaire, enquête internationale, Cour pénale internationale, lutte contre l'impunité, accords de paix, failed state. 


\section{AUTEUR}

JELENA APARAC

Doctorante en droit international public, ATER à l'Université Paris Ouest Nanterre La Défense, chargée d'enseignement en droit international humanitaire, droit international public et libertés fondamentales. 